\title{
Capacidade Antioxidante de Sucos e Néctares de Uvas do Rio Grande do Sul
}

\author{
Gilvane Bassanesi' ; Luciana Bavaresco Andrade Touguinha'; Mirian Salvador ${ }^{2}$
}

\gilbassanesi@yahoo.com.br

1. Universidade de Caxias do Sul, Caxias do Sul /RS.

2. Instituto de Biotecnologia - Universidade de Caxias do Sul, Caxias do Sul /RS.

\section{Histórico do Artigo:}

Recebido em: 30 de janeiro de $2020 \quad$ Aceito em: 20 de julho de $2020 \quad$ Publicado em: 31 de agosto de 2020

Resumo: 0 suco de uva é um produto amplamente consumido por suas características nutricionais e presença de compostos fenólicos, os quais possuem importante atividade antioxidante. Dessa maneira, o objetivo deste estudo foi avaliar a atividade antioxidante e o teor de compostos fenólicos totais de diferentes tipos de sucos de uva e néctares, encontrados no comércio de duas cidades do Rio Grande do Sul. Foram selecionados 17 sucos de uva tintos, 06 sucos brancos e 4 néctares, representando a totalidade das amostras encontradas em mercados das duas cidades. A atividade antioxidante foi determinada pela capacidade de varredura do radical livre DPPH $(2,2$-difenil1-picrilhidrazil) e o teor de compostos fenólicos totais (TPT) foi avaliado pelo método de Folin-Ciocalteau. A atividade antioxidante variou de 50,14 a 97,38\% e o TPT variou de 143,83 a 1.113,83 mgEAG/mL, considerando sucos e néctares. A maior atividade antioxidante foi encontrada nos sucos tintos, seguidos dos sucos brancos e néctares. Os maiores valores de TPT foram encontrados nos sucos tintos, seguidos dos néctares e sucos brancos. Este estudo colabora com o conhecimento do teor de TPT e atividade antioxidante de sucos produzidos na região Nordeste do estado do Rio Grande do Sul.

Palavras-chave: Compostos fenólicos, DPPH, Saúde humana, Vitis sp.

\section{Antioxidant Capacity of Grape Juices and Nectars from Rio Grande do Sul}

\begin{abstract}
Grape juice is a product widely consumed for its nutritional characteristics and presence of phenolic compounds, which have important antioxidant activity. Thus, the objective of this study was to evaluate the antioxidant activity and the total phenolic compounds content of different types of grape juice and nectars, found in commerce in two cities in Rio Grande do Sul. We selected 17 red grape juices, 6 white grape juices and 4 nectars, representing all the samples found in markets of the two cities. The antioxidant activity was determined by the free radical scavenging ability using the DPPH (2,2-diphenyl-1-picrylhydrazyl) method, and content the of total phenolic compounds (TPC) was evaluated by the Folin-Ciocalteau method. For comparative analysis of the data, variance analysis and Students t-test for independent samples were applied. The antioxidant activity ranged from 50.14 to $97.38 \%$ and the TPC ranged from 143.83 to $1111.83 \mathrm{mgEAG} / \mathrm{mL}$. The highest antioxidant activity was found in red juices, followed by white juices and nectars. The highest values of TPC were found in red juices, followed by nectars and white juices. This study contributes to the knowledge about TPC and biological activity of juices produced in the Northeast region of the state of Rio Grande do Sul.
\end{abstract}

Keywords: Phenolic compounds, DPPH, Human health, Vitis sp. 


\section{Capacidad Antioxidante de Jugos de Uva y Néctares de Rio Grande do Sul}

Resumen: El jugo de uva es un producto ampliamente consumido por sus características nutricionales y la presencia de compuestos fenólicos, que tienen una importante actividad antioxidante. Por lo tanto, el objetivo de este estudio fue evaluar la actividad antioxidante y el contenido de compuestos fenólicos totales de diferentes tipos de zumos y néctares de uva, encontrados en el comercio en dos ciudades en Rio Grande do Sul. Se seleccionaron 17 jugos de uva roja, 06 jugos blancos y 4 néctares, lo que representa la totalidad de las muestras encontradas en los mercados de ambas ciudades. La actividad antioxidante se determinó por la capacidad de escanear el radical libre DPPH • (2,2-difenil-1-picrilhidrazil) y el contenido de compuestos fenólicos totales (TPT) se evaluó mediante el método Folin-Ciocalteau. La actividad antioxidante varió de 50.14 a $97.38 \%$ y TPT varió de 143.83 a 1,113.83 mgEAG / mL, considerando los jugos y néctares. La mayor actividad antioxidante se encontró en los jugos rojos, seguidos de los jugos blancos y néctares. Los valores más altos de TPT se encontraron en jugos rojos, seguidos de néctares y jugos blancos. Este estudio colabora con el conocimiento del contenido de TPT y la actividad antioxidante de los jugos producidos en la región noreste del estado de Rio Grande do Sul.

Palabras clave: Compuestos fenólicos, DPPH, Salud humana, Vitis sp.

\section{INTRODUÇão}

A produção do suco de uva no Brasil é de grande importância econômica e social (IRITTI e FAOR0, 2009). 0 consumo tem aumentado pela qualidade do sabor e pelos benefícios que ele traz para a saúde humana (PONTES et al., 2010). Caracterizada pelo clima úmido e pelo solo basáltico, a Serra Gaúcha se beneficia da alta produtividade, sem necessidade de irrigação, e 0 resultado dessa combinação reflete na qualidade das uvas capazes de gerar um suco de coloração púrpura-violácea intensa, aroma marcante e excelente equilíbrio entre doçura e acidez (LEAL et al., 2017). Com base na safra 2018, pode-se dizer que a Serra Gaúcha é responsável por aproximadamente 95\% do suco elaborado no estado do Rio Grande do Sul. Na Serra Gaúcha foram produzidos 32,7 milhões de litros de suco elaborados na última safra e 34,4 milhões de litros no Rio Grande do Sul (IBRAVIN, 2019).

Segundo a legislação brasileira, o suco pode ser classificado: (1) suco de uva integral, onde não é permitida a adição de açúcares; (2) suco de uva concentrado, o qual é parcialmente desidratado, devendo apresentar no mínimo $65^{\circ}$ Brix de sólidos solúveis totais; (3) suco de uva desidratado obtido a partir da fase sólida resultante da desidratação do suco de uva, podendo apresentar somente 3\% de umidade; (4) suco de uva adoçado com açúcar; (5) suco de uva processado, obtido através da diluição do suco concentrado ou desidratado (GUERRA, 2016). Já o néctar de uva é a bebida que contém, no mínimo, 50\% de suco da fruta não fermentada, obtida a partir da diluição em água da parte comestível da fruta ou de seu extrato, com adição de açúcares (BRASIL, 2009). 
0 suco de uva brasileiro é elaborado principalmente com uvas dos grupos das Vitis labrusca, conhecidas como americanas e híbridas, tintas, principalmente Bordô, Isabel e Concord (B0AS et al., 2017). No entanto, têm sido utilizadas também uvas das variedades Niágara Branca e Moscato para produção de sucos brancos (RIZZ0N et al., 1998).

A produção de uvas pode ser de origem agrícola convencional, a qual utiliza herbicidas, inseticidas e fungicidas, principalmente dos grupos químicos ditiocarbamatos e organofosforados no seu cultivo (DANI et al., 2007). Já no cultivo orgânico as uvas colhidas dos vinhedos não contêm uso de fitodefensivos, onde a produção é conhecida por ser um sistema sustentável (FREITAS et al., 2010). Neste manejo as uvas são produzidas através de uma agricultura vista pela sociedade como mais justa, além de ser economicamente viável e ecologicamente correta (SOUZA et al., 2018). Os alimentos orgânicos podem ser considerados de maior qualidade por serem cultivados em solos com maior equilíbrio de nutrientes, e por sua produção não conter agrotóxicos e fertilizantes sintéticos (B0AS et al., 2014). Com isso, a agricultura orgânica, além de não causar danos ao meio ambiente, é de extrema importância para a saúde humana (FAGUNDES e SCHEREIBER, 2017).

Estudos realizados por Dani et al. (2007) com amostras de sucos de uva brancos Niágara e tintos Bordô, orgânicos e convencionais, mostraram que os sucos elaborados com uvas provenientes de cultivos orgânicos apresentam um teor de compostos fenólicos maior que o suco produzido a partir de uvas de cultivos convencionais (CARNEIR0 et al., 2013). Isto pode ocorrer pois os compostos fenólicos são metabólitos secundários das plantas, produzidos e acumulados durante situações de estresse da planta. Como os pesticidas não são utilizados na agricultura orgânica, as plantas tornam-se mais suscetíveis à ação de patógenos e doenças, produzindo assim uma maior quantidade desses compostos com a finalidade de proporcionar a defesa da planta contra estes agressores (0LSSON et al., 2013; MARGRAF et al., 2016).

0s radicais livres em excesso causam estresse oxidativo, o qual está associado a doenças cardiovasculares, câncer, diabetes mellitus e hipertensão (ACHKAR et al., 2013). Antioxidantes, incluindo os compostos fenólicos, podem reduzir este risco de dano oxidativo através da eliminação desses radicais livres (B0AS et al., 2017). Dentre as frutas, as uvas são fontes importantíssimas de compostos fenólicos com propriedades medicinais (BURIN et al., 2010). Compostos fenólicos têm demonstrado que podem prevenir o estresse oxidativo, oxidação da lipoproteína de baixa densidade (LDL-c) (DAY et al., 1997), agregação plaquetária (OSMAN et al., 1998), danos ao DNA (FRANKEL et al.,1998), além da melhora na proteção contra episódios aterotrombóticos e doenças coronarianas (SINGLETARY et al., 2003). 
Desta forma, o objetivo do presente estudo foi avaliar a atividade antioxidante e o teor de compostos fenólicos de diferentes variedades de sucos de cultivo orgânico e convencional e néctares de uva, disponíveis para a população no comércio da cidade de Caxias do Sul e Flores da Cunha, região serrana do Rio Grande do Sul.

\section{MATERIAL E MÉTODOS}

\section{Amostras}

Para este estudo foram adquiridos 23 sucos de uva integrais (3 de cultivo orgânico e 20 de cultivo convencional) e 4 néctares de uva (um de cultivo orgânico e 3 de cultivo convencional) representando a totalidade de amostras disponíveis no comércio e em vinículas das cidades de Caxias do Sul e Flores da Cunha/RS. A coleta das amostras foi realizada no mês de março de 2019. 0 critério utilizado para a escolha das amostras foi que todas as amostras fossem de suco integral (100\% uva) e que a rotulagem fornecesse o maior número de informações possíveis como tipo de uvas utilizadas e se era de origem de cultivo orgânico ou convencional que foi o foco desse estudo. Todas as amostras proveram de garrafas de vidro e foram armazenadas no Laboratório de Estresse 0xidativo e Antioxidantes a temperatura ambiente e abrigo da luz até a realização dos ensaios, nesse dia foram abertas e imediatamente submetidas aos ensaios de atividade antioxidante. Aliquotas de $50 \mathrm{~mL}$ foram armazenadas de cada suco em freezer $-20^{\circ} \mathrm{C}$ caso fosse necessária a repetição dos ensaios.

\section{Atividade antioxidante}

A atividade antioxidante dos sucos de uva foi determinada através do teste de capacidade de varredura do radical livre DPPH ${ }^{\bullet}$ (2,2-difenil-1-picrilhidrazil), conforme

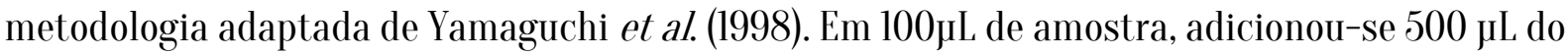
reagente $\mathrm{DPPH} \bullet$ e $400 \mu \mathrm{L}$ de tampão Tris- $\mathrm{HCl}(100 \mathrm{mM}, \mathrm{pH}=7,0)$. As amostras foram agitadas e permaneceram em repouso e ao abrigo da luz por 20 minutos. Após, as absorbâncias foram determinadas a $517 \mathrm{~nm}$, em espectrofotômetro (UV-1700, Shimadzu Pharmaspec). 0 radical livre DPPH• quando estável possui coloração violeta e na presença de substâncias antioxidantes é reduzido a coloração amarela. A mudança de cor é monitorada por espectrofotometria. 0s resultados foram expressos em percentual de varredura, ou seja, a capacidade da amostra em reduzir a atividade do radical livre $\mathrm{DPPH}^{\bullet}$ 


\section{Teor de compostos fenólicos totais}

0 teor de compostos fenólicos totais das amostras foi determinado pelo método colorimétrico de Folin-Ciocalteau, com modificações (SINGLETON; ROSSI, 1965). Em $100 \mu \mathrm{L}$ de amostra, adicionou-se $500 \mu \mathrm{L}$ de reagente Folin-Ciocalteau $1 \mathrm{~N}$ e $400 \mu \mathrm{L}$ de carbonato de cálcio $\left(\mathrm{Na}_{2} \mathrm{CO}_{3}\right) 7,5 \%$. Para a curva padrão, utilizou-se ácido gálico em concentrações que variaram de 0,025 a $0,75 \mathrm{mg} / \mathrm{mL}$. As amostras foram agitadas e permaneceram em repouso e ao abrigo da luz pelo período de 30 minutos. As absorbâncias foram determinadas a $765 \mathrm{~nm}$, em espectrofotômetro (UV-1700, Shimadzu Pharmaspec). 0s resultados foram expressos em mg de equivalentes de ácido gálico $(\mathrm{EAG} / \mathrm{mL})$ de suco.

\section{Análise estatística}

Foram realizados dois testes independentes para cada ensaio com duplicata para cada amostra analisada. Os resultados foram apresentados como médias e desvio padrão. Para análise comparativa dos dados foi aplicada a análise de variância (ANOVA) para amostras independentes e pós teste de Tukey $(\mathrm{p}<0,05)$. 0 coeficiente de correlação de Pearson foi utilizado para correlacionar a atividade antioxidante e o teor de compostos fenólicos totais dos sucos e néctares de uva analisados. 0 nível de significância aceito foi de $\mathrm{p}<0,05$. Para realização das análises foi utilizado o programa Statistical Package for the Social Sciences (SPSS®) versão 22.0.

\section{RESULTADOS E DISCUSSÃO}

Dos diferentes sucos de uva utilizados para a realização do foram selecionadas 27 amostras, produzidas por 18 vinícolas diferentes da região da Serra Gaúcha, porém sem especificação de safra. As uvas utilizadas foram Isabel, Bordô, Concord, Niágara Branca, Moscato e Lorena. Foram selecionados 3 sucos e 1 néctar provenientes de cultivo orgânico (amostras 1, 2, 3 e 24) e 23 convencionais.

Para a análise da atividade antioxidante, utilizou-se o método de inibição do radical DPPH $•$ Esse método é um dos mais utilizados para avaliar a atividade antioxidante de sucos de uva por ser um ensaio com boa reprodutibilidade e de fácil aplicação e execução (DUARTE et al., 2006).

Os resultados obtidos no ensaio que determinou a atividade antioxidante dos diferentes tipos de sucos de uva estão expostos na Tabela 1. As porcentagens de varredura variaram de $50,14 \%$ (amostra 27) a 97,38\% (amostra 15). 0s maiores valores foram encontrados nas amostras 
de sucos de uva tinto integral, seguido de sucos brancos e néctares (Figura 1 e 2). Não foram observadas diferenças significativas estatisticamente entre as amostras de sucos tintos. Todos os sucos brancos apresentaram a mesma atividade antioxidante. As atividades antioxidantes dos néctares variaram entre si, provavelmente devido a maior ou menor adição de água durante o seu preparo (MEL0 et al., 2016).

0s sucos brancos apresentaram maior atividade antioxidante do que os néctares, mesmo tendo um teor de TPT menor do que os néctares. Este fato pode ser explicado pelas diferentes classes de compostos fenólicos encontrados em uvas brancas e tintas. Outro fator a ser considerado é a presença de ácido ascórbico em uvas brancas. Não foram encontrados, até o momento, dados sobre o teor de ácido ascórbico de sucos de uva ou uvas V. labrusca. No entanto, sabe-se que a uva Itália tem um conteúdo maior de ácido ascórbico do que a uva rubi (NEPA, 2011).

Desta forma, pode-se pensar que o ácido ascórbico dos sucos de uva brancos também tenha contribuído para a atividade antioxidante observada no presente trabalho. Estudos adicionais com um maior número de amostras e incluindo a identificação dos compostos fenólicos e vitamina C seriam importantes para esclarecer este resultado.

Tabela 1 - Atividade antioxidante avaliada por DPPH• e TPT de sucos e néctares de uva.

\begin{tabular}{|c|c|c|c|c|c|}
\hline Amostra & Marca & Tipo de uva & $\begin{array}{c}\text { Convencional/ } \\
\text { Orgânico } \\
\end{array}$ & $\begin{array}{c}\mathrm{DPPH}^{\bullet} \\
\text { (\% varredura) }\end{array}$ & TPT (mgEAG/mL) \\
\hline \multicolumn{6}{|c|}{ Integral Tinto } \\
\hline 1 & $\mathrm{~A}$ & Isabel e Bordô & Orgânico & $97,13 \pm 0,10^{\mathrm{a}}$ & $723,83 \pm 2,93^{\text {fgh }}$ \\
\hline 2 & B & Bordô & Orgânico & $97,21 \pm 0,06^{\mathrm{a}}$ & $1113,49 \pm 0,01^{\mathrm{a}}$ \\
\hline 3 & $\mathrm{C}$ & Isabel e Bordô & Orgânico & $97,23 \pm 0,01^{\mathrm{a}}$ & $880,39 \pm 3,90^{\text {cde }}$ \\
\hline 4 & A & Isabel & Convencional & $96,59 \pm 0,03^{\mathrm{a}}$ & $780,39 \pm 2,93^{\text {efg }}$ \\
\hline 5 & D & Isabel e Bordô & Convencional & $96,44 \pm 0,13^{a}$ & $811,42 \pm 7,56^{\mathrm{def}}$ \\
\hline 6 & $\mathrm{E}$ & Bordô & Convencional & $97,35 \pm 0,10^{\mathrm{a}}$ & $670,04 \pm, 98^{\mathrm{gh}}$ \\
\hline 7 & $\mathrm{~F}$ & Niágara & Convencional & $96,60 \pm 0,17^{\mathrm{a}}$ & $500,39 \pm 65,35^{\mathrm{jk}}$ \\
\hline 8 & G & Niágara & Convencional & $97,16 \pm 0,01^{\mathrm{a}}$ & $812,11 \pm 1,69^{\mathrm{def}}$ \\
\hline 9 & $\mathrm{H}$ & Bordô & Convencional & $97,24 \pm 0,07^{\mathrm{a}}$ & $1003,83 \pm 22,43^{\mathrm{ab}}$ \\
\hline 10 & I & Isabel e Concord & Convencional & $97,13 \pm 0,03^{\mathrm{a}}$ & $955,56 \pm 10,73^{\mathrm{bc}}$ \\
\hline 11 & $J$ & Niágara & Convencional & $97,19 \pm 0,02^{\mathrm{a}}$ & $937,63 \pm 69,25^{\mathrm{bc}}$ \\
\hline 12 & K & Niágara & Convencional & $97,20 \pm 0,03^{\mathrm{a}}$ & $907,28 \pm 53,64^{\text {bcd }}$ \\
\hline 13 & $\mathrm{~L}$ & Niágara & Convencional & $96,93 \pm 0,10^{\mathrm{a}}$ & $750,04 \pm 0,98^{\mathrm{fg}}$ \\
\hline 14 & M & Isabel e Bordô & Convencional & $97,07 \pm 0,01^{\mathrm{a}}$ & $879,70 \pm 4,88^{\text {cde }}$ \\
\hline 15 & $\mathrm{~N}$ & $\begin{array}{l}\text { Isabel, Bordô e } \\
\text { Concord }\end{array}$ & Convencional & $97,38 \pm 0,02^{\mathrm{a}}$ & $922,46 \pm 0,98^{\mathrm{bcd}}$ \\
\hline 16 & 0 & Niágara & Convencional & $97,10 \pm 0,05^{\mathrm{a}}$ & $772,11 \pm 4,88^{\mathrm{efg}}$ \\
\hline 17 & $\mathrm{P}$ & Niágara & Convencional & $95,85 \pm 0,58^{\mathrm{a}}$ & $617,63 \pm 0,23^{h i}$ \\
\hline \multicolumn{6}{|c|}{ Integral branco } \\
\hline 18 & A & $\begin{array}{c}\text { Niágara Branca e } \\
\text { Moscato }\end{array}$ & Convencional & $86,67 \pm 1,16^{b}$ & $149,35 \pm 5,85^{\mathrm{n}}$ \\
\hline 19 & D & Niágara Branca & Convencional & $86,45 \pm 0,85^{\mathrm{b}}$ & $224,52 \pm 6,58^{\mathrm{mn}}$ \\
\hline 20 & $\mathrm{E}$ & $\begin{array}{c}\text { Niágara Branca e } \\
\text { Moscato }\end{array}$ & Convencional & $87,21 \pm 2,01^{\mathrm{b}}$ & $323,83 \pm 10,73^{\mathrm{lm}}$ \\
\hline
\end{tabular}


Capacidade Antioxidante de Sucos e Néctares de Uvas do Rio Grande do Sul

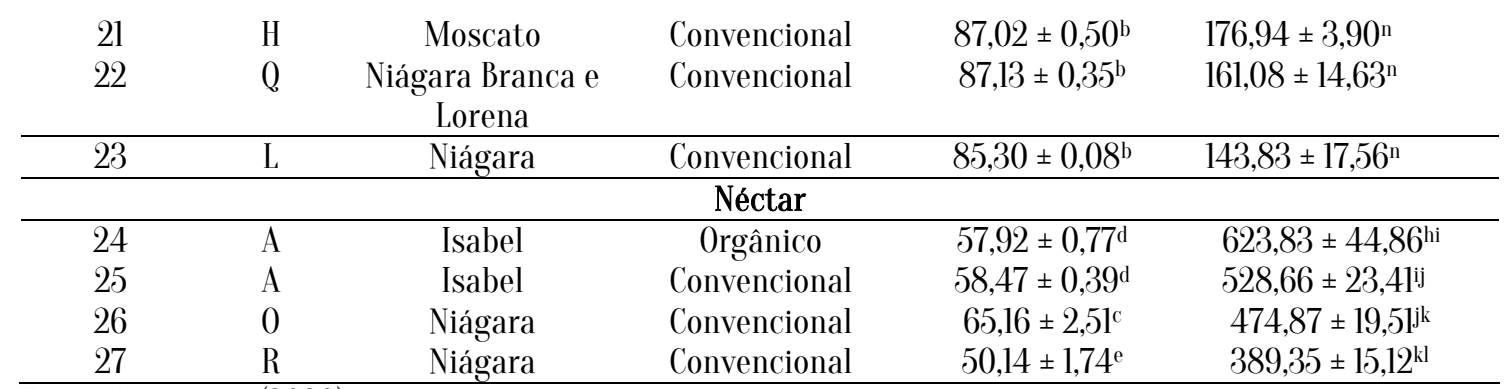

Fonte: Bassanesi (2020)

Rodrigues et al., (2019) analisaram dez sucos de uva integrais, oito de uvas tintas e dois de uvas brancas, encontrando que os sucos tintos apresentaram maior atividade antioxidante do que os brancos. Resultados semelhantes foram descritos também por Vargas et al. (2008), que utilizaram 8 amostras de sucos integrais, sete tintos e um branco, coletados em estados diferentes do Brasil. 0 valor do percentual de varredura dos sucos tintos foi semelhante a encontrada neste trabalho.

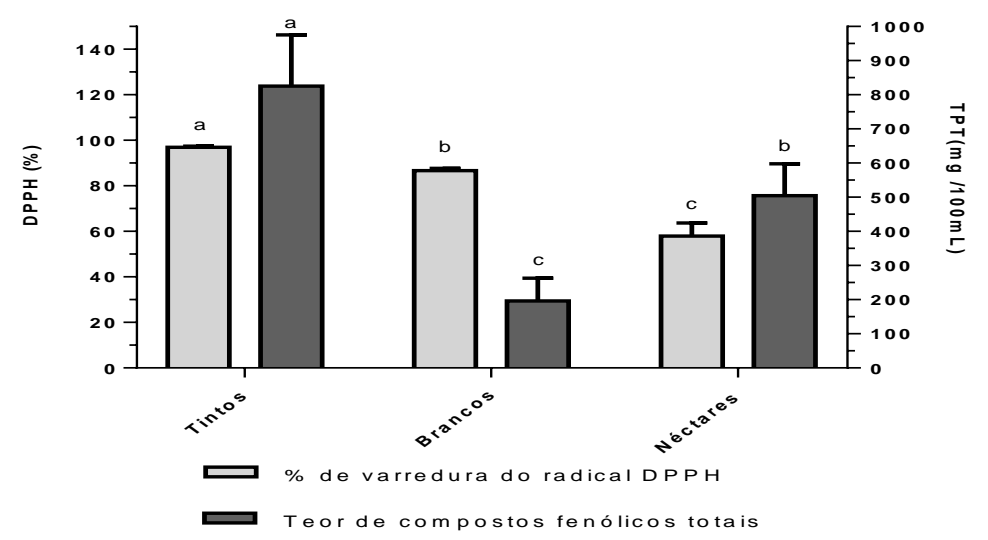

Figura 1 - Valores e médias da atividade antioxidante (DPPH•) e teor de compostos fenólicos totais (TPT) dos sucos de uva e néctar.

Fonte: Bassanesi (2020) 


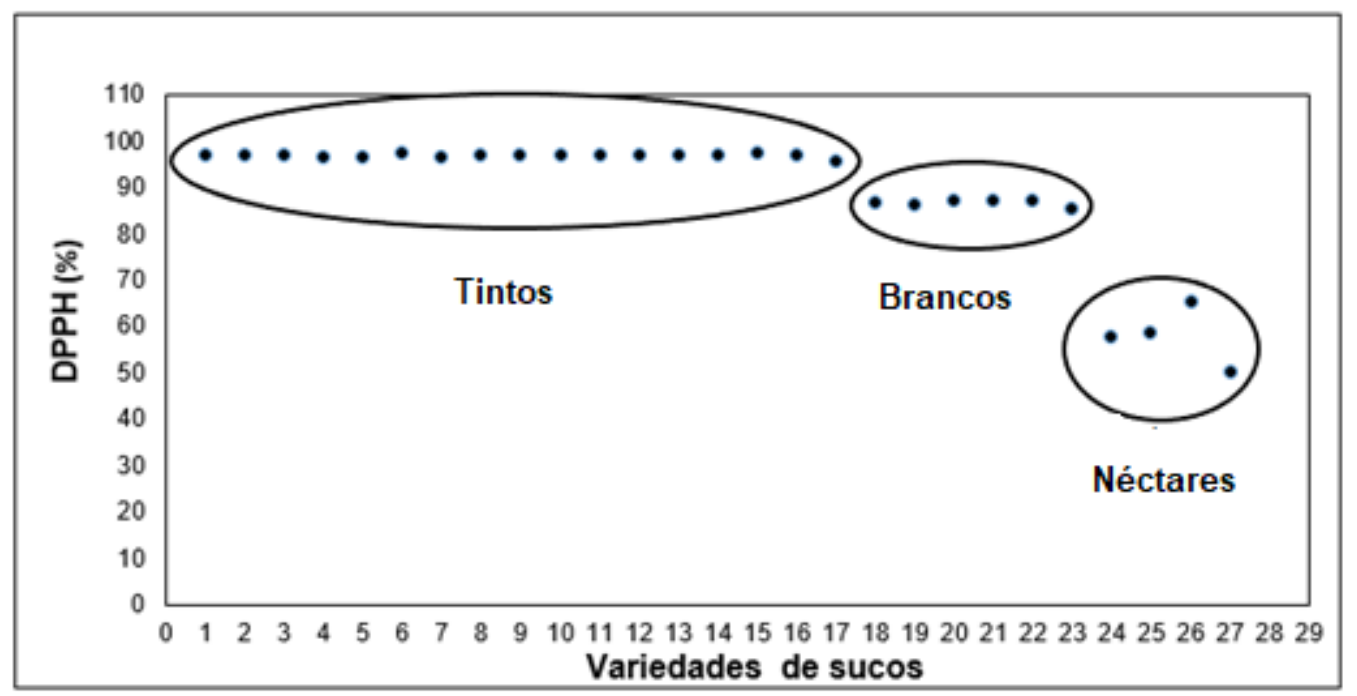

Figura 2 - Distribuição das amostras de sucos de uva e néctares analisados no que se refere ao perfil da atividade antioxidante.

Fonte: Bassanesi (2020)

Os resultados obtidos no ensaio que determinou TPT nos diferentes tipos de sucos de uva analisados estão apresentados na Tabela 1. 0 conteúdo de compostos fenólicos totais variou de 143,83 mgEAG/mL (amostra 23) a 1113,49 mgEAG/mL (amostra 2). 0s maiores resultados de TPT foram encontrados nos sucos de uva tintos integrais, seguido de néctares e após de sucos brancos (Figura 2 e 3).

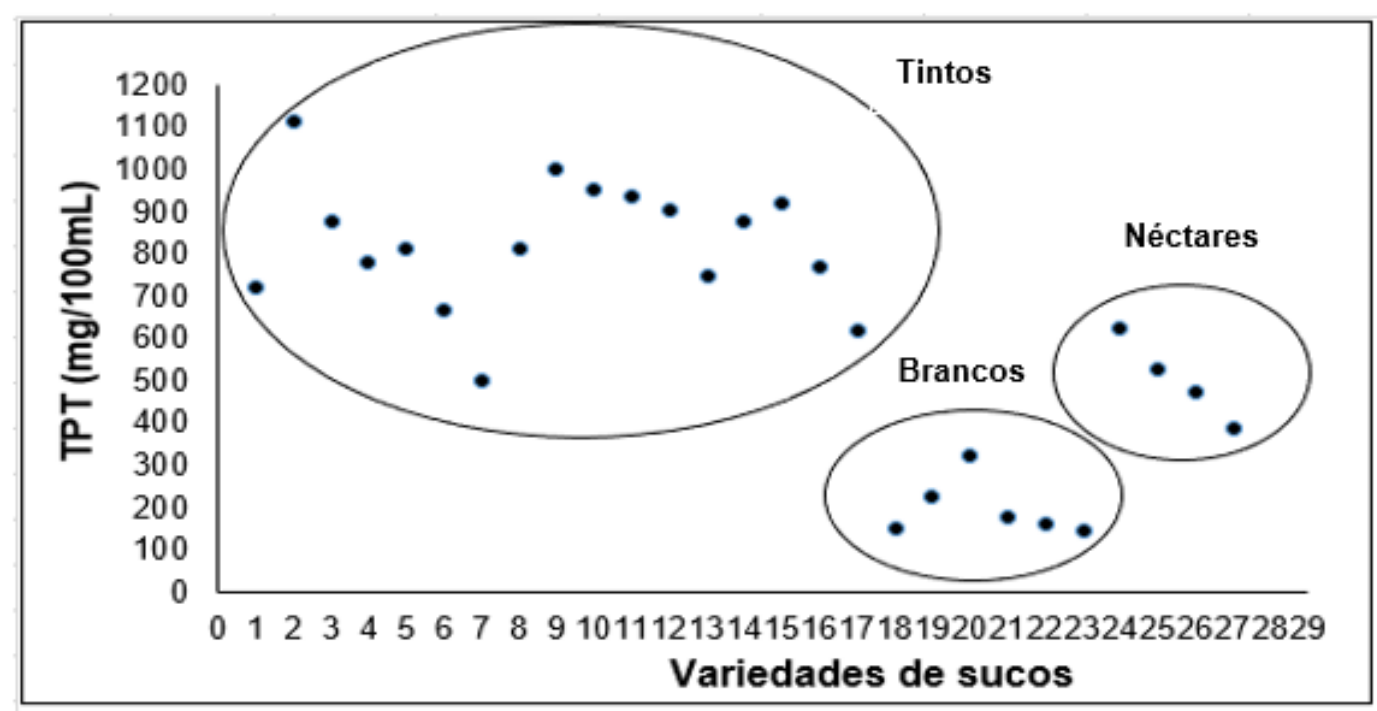

Figura 3. Distribuição das amostras de sucos de uva e néctares analisados em relação ao teor de compostos fenólicos totais.

Fonte: Bassanesi (2020)

Observou-se uma correlação positiva moderada entre o teor de compostos fenólicos e atividade antioxidante para sucos tintos $(r=0,527 ; \mathrm{p}=0,001)$. Por outro lado, não houve 
correlação significativa entre teor de compostos fenólicos e atividade antioxidante para sucos brancos $(r=0,366 ; p=0,242)$. Para os néctares a correlação do teor de compostos fenólicos e atividade antioxidante foi muito baixa $(r=0,360 ; p=0,381)$.

Comparando as amostras 1 e 3 (orgânicas, produzidas a partir das variedades Isabel e Bordô), com as amostras 5 e 14 (convencionais, produzidas a partir das variedades Isabel e Bordô), não foram observadas diferenças na capacidade antioxidante e teor de compostos fenólicos totais (Tabela 1). Da mesma forma, comparando a amostra de néctar 24 (orgânico, produzido a partir da variedade de uva Isabel) com a amostra 25 (convencional, produzido a partir da variedade de uva Isabel) também não foi observada diferença na atividade antioxidante e compostos fenólicos totais. Dessa forma não foi evidenciada influência do cultivo orgânico na atividade antioxidante e compostos fenólicos totais. No entanto, ensaios com um número maior de amostras são necessários para poder avaliar esta variável.

Dani et al. (2007) realizaram o primeiro estudo que mostrou a atividade antioxidante de sucos de uva produzidos com variedades de V. labrusca (Bordô e Niágara), orgânicos e convencionais. 0 estudo demonstrou que os sucos orgânicos apresentaram maior atividade antioxidante, não observado no presente trabalho.

Sucos de uva são produtos alimentares ricos em compostos fenólicos (POLONIO et al., 2014). 0 consumo da uva e seus derivados estão associados a vários efeitos benéficos para a saúde, como: aumento da capacidade antioxidante, redução de lipoproteínas oxidadas de baixa densidade (LDL) (BOAS et al., 2017), aumento de lipoproteínas de alta densidade (HDL), diminuição da agregação plaquetária, melhora da capacidade cardiovascular (EVANS et al., 2014). Um dos mecanismos pelos quais os compostos fenólicos atuam é através da redução das espécies reativas de oxigênio e de nitrogênio, as quais estão relacionadas ao desenvolvimento das doenças cardiovasculares (ROLDAN, 2016).

Os principais compostos fenólicos encontrados na uva são os flavonóides (antocianinas, flavanóis e flavonóis), os estilbenos (resveratrol), os ácidos fenólicos (derivados dos ácidos cinâmicos e benzóicos) e uma grande variedade de taninos (COSME et al., 2018). No entanto, a quantidade e o tipo de compostos fenólicos totais não são necessariamente os mesmos em diferentes tipos de uva (FULEKI e SILVA, 2003). Os conteúdos de compostos fenólicos totais e de antocianinas nas uvas variam de acordo com a espécie, variedade, maturidade, condições climáticas e a forma do seu cultivo (DANI et al.;2007; ACHKAR et al., 2013). Além disso, o processo ao qual a uva e o mosto são submetidos durante a produção do suco, tais como tipo de extração, tempo de contato entre o suco e as partes sólidas da uva (casca e sementes), prensagem, tratamentos térmicos, tratamentos enzimáticos e adição de dióxido de enxofre e ácido tartárico 
também podem interferir na quantidade de compostos fenólicos totais no suco de uva (MALACRIDA e MOTTA, 2005; T0ALD0, 2015).

Sabe-se que antocianinas são pigmentos vegetais que dão cor a uva, ou seja, uvas tintas contém uma quantidade maior de antocianinas. Boas et al., (2014) realizaram um estudo onde encontraram maiores níveis de compostos fenólicos totais em sucos de uvas tintas. Estes dados sugerem que as antocianidinas exercem importante papel na atividade antioxidante dos sucos tintos.

\section{CONSIDERAÇÕES FINAIS}

Todos os sucos e néctares de uva analisados apresentaram presença de compostos fenólicos totais e atividade antioxidante. 0 maior TPT foi encontrado nos sucos tintos seguido dos néctares e sucos brancos. A maior atividade antioxidante foi observada nos sucos tintos, seguido dos sucos brancos e néctares. Não foram evidenciadas influência do cultivo orgânico na atividade antioxidante e teor de polifenóis totais para este estudo, porém ensaios com um número maior de amostras seriam necessários para avaliar estas variáveis.

\section{AGRADECIMENTOS}

LE0A (Laboratório de Estresse 0xidativo e Antioxidantes/UCS), CNPq (Conselho Nacional de Desenvolvimento Científico e Tecnológico), FAPERGS (Fundação de Amparo à Pesquisa do Estado do Rio Grande do Sul).

\section{REFERÊNCIAS BIBLIOGRÁFICAS}

ACHKAR, M.T.; NOVAES, G.M.; SILVA, M.G.D.; VILEGAS, W. Propriedade antioxidante de compostos fenólicos: importância na dieta e na conservação de alimentos. Revista da Universidade Vale do Rio Verde, v. 11, n. 2, p. 398406, 2013. Disponível em: https://repositorio.unesp.br/bitstream/handle/11449/123439/ISSN2236-5362-2013-1102-398-406.pdf? sequence=1GisAllowed=y

BOAS, A.C.V.; HENRIQUE, P.C.; OLIVEIRA LIMA, L.C.; NETO, A.D. Antioxidant activity, anthocyanins and organic acids content of grape juices produced in Southwest of Minas Gerais, Brazil. Ciência e Agrotecnologia, v. 38, n. 5, p. 480486, 2014. Disponível em: http://www.scielo.br/pdf/cagro/v38n5/a07v38n5.pdf

BOAS, A.C.V.; NASSUR, R.C.M.R.; FREIRE, J.M.; DECARLOS A.N.; LIMA, L.C.O. Quality characteristics and antioxidant activity of juices produced with irradiated grapes. Revista Brasileira de Fruticultura, v. 39, n. 2, p. 1- 11, 2017. Disponível em: http://www.scielo.br/pdf/rbf/v39n2/0100-2945-rbf-39-2-e-188.pdf 
BRASIL. Constituição. Decreto no 6.871, de 4 de junho de 2009. Regulamenta a Lei no 8.918, de 14 de julho de 1994 , que dispõe sobre a padronização, a classificação, o registro, a inspeção, a produção e a fiscalização de bebidas. Brasília. 2009.

BURIN, M.V.; FALCÃO, Lila D.; GONZAGA, L.V.; FETT, R.; ROSIER, J.P.; BORDGNON, L.T. Colour, phenolic content and antioxidant activity of grape juice. Ciência e Tecnologia de Alimentos, v. 30, n. 4, p. 1027- 1032, 2010.Disponível em: http://www.scielo.br/pdf/cta/v30n4/v30n4a30.pdf

CARNEIR0, A.P.G.; ABREU, D.A.; SOARES, D.J.; COSTA, E.A.; SILVA, L.M.R.; BARBOSA, L. C.; SOUSA, P.H.M.; FIGUEIRED0, R.W. Avaliação da rotulagem, caracterização química, físico-química e reológica de néctares de uva comercializados na cidade de Fortaleza - CE. Alimentos e Nutrição, v. 24, n. 2, p. 241-249, 2013. Disponível em: http://servbib.fcfar.unesp.br/seer/index.php/alimentos/article/viewFile/241/2263

COSME, F.; PINT0, T.; VILELA, A. Phenolic Compounds and Antioxidant Activity in Grape Juices: A Chemical and Sensory View. Beverages, v. 4, n. 1, p.22-36, 2018. Disponível em: https://www.mdpi.com/2306-5710/4/1/22

DANI, C.; OLIBONI, L.S.; VANDERLINDE, R.; BONATTO, D.; SALVADOR, M.; HENRIQUES, J.A.P. Phenolic content and antioxidant activities of white and purple juices manufactured with organically- or conventionally-produced grapes.Journal of Medicinal Food, v. 45, n. 12, p. 2574-2580, 2007. Disponível em: https://www.ncbi.nlm.nih.gov/pubmed/17683842

DAY, A.P., KEMP, H.J., BOLTON, C., HARTOG, M., STANSBIE, D. Effect of concentrated red grape juice consumption on serum antioxidant capacity and low-density lipoprotein oxidation. Annais Nutrition of Metabolism, v. 41, n. 6 , p. 353-357, 1997. Disponível em: https://www.ncbi.nlm.nih.gov/pubmed/9491190

DUARTE, J.M.; SANTOS, R.J.; GENOVESE, M.I.; LAJOLO, F.M. Avaliação da atividade antioxidante utilizando sistema ß-caroteno/ácido linoleico e método de sequestro de radicais DPPH. Ciência Tecnologia Alimentos, v. 26, n. 2, p. 446-452, 2006. Disponível em: http://www.scielo.br/pdf/cta/v26n2/30196.pdf

EVANS, M.; WILSON, D.; GUTHRIE, N. A randomized, double-blind, placebo-controlled, pilot study to evaluate the effect of whole grape extract on antioxidant status and lipid profile, Journal of Functional Foods, v.7, n.1, p. 680691, 2014. Disponível em: https://www.sciencedirect.com/science/article/pii/S1756464613003113?via\%3Dihub

FAGUNDES, C:; SCHEREIBER, D. Concepção de um modelo para avaliação da sustentabilidade no processo produtivo do suco de uva orgânico. Desenvolvimento e Meio Ambiente, v. 41, n. 2, p. 57-82, 2017. Disponível em: https://revistas.ufpr.br/made/article/view/49766/33403

FRANKEL, E.N.; BOSANEK, C.A.; MEYER, A.S.; SILLIMAN, K.; KIRK, L.L., Commercial grape Juices inhibit the in vitro oxidation of human lowdensity lipoproteins. Journal Agriculture and Food Chemistry, v. 46, n. 2, p. 834-838, 1998. Disponível em: https://pubs.acs.org/doi/pdf/10.1021/jf9707952\#

FREITAS, A.F.; DETONI, A.M.; CLEMENTE, E.; OLIVEIRA, C.C. Determinação de resveratrol e características químicas em sucos de uvas produzidas em sistemas orgânico e convencional. Revista Ceres, v. 57, n. 1, p.1-5, 2010. Disponível em: http://www.scielo.br/pdf/rceres/v57nl/a01v57nl.pdf

FULEKI, T., SILVA, R.J.M. Effects of cultivar and processing method on the contents of catechins and procyanidins in grape juice. Journal Agriculture and Food Chemistry, v. 51, n.1, p. 640-646, 2003. Disponível em: https://www.ncbi.nlm.nih.gov/pubmed/12537435

GUERRA, Celito Crivellaro. Sistema para Elaboração de Suco de Uva Integral em Pequenos Volumes: Suquificador Integral. Bento Gonçalves: Embrapa, $2016 . \quad 32 \quad$ p. $\quad$ Disponível em: https://ainfo.cnptia.embrapa.br/digital/bitstream/item/141246/1/doc96.pdf.

INSTITUTO BRASILEIRO DO VINHO (IBRAVIN-RS). Nem tudo que tem uva é SUC0: Saiba qual a diferença entre o verdadeiro suco de uva e as outras bebidas. Disponível em: http://www.ibravin.org.br/admin/arquivos/downloads/1455901932.pdf.

IRITTI, M.; FA0R0, F. Bioactivity of grape chemicals for human health. Natural Product Communications, v. 4, n. 5, p. 61l-634, 2009. Disponível em: https://journals.sagepub.com/doi/pdf/10.1177/1934578X0900400502 
LEAL, J.B.; CARVALHO, F.0.; GONÇALVES, D.C., LEAL, J.B., SILVA, G.C.L.; CARNEVALI, L.C.J.; HOEFEL, A.L. Resveratrol: composição química e seus benefícios a saúde. Revista Brasileira de Obesidade, Nutrição e Emagrecimento, v. 67.n. 11, p. 620-629. 2017. Disponível em: http://www.rbone.com.br/index.php/rbone/article/view/598/484

MALACRIDA, C.R.; MOTTA, S. Compostos fenólicos totais e antocianinas em suco de uva: Total phenolics and anthocyanins in grape juice. Ciência e Tecnologia de Alimentos, v. 25, n. 4, p. 659 - 664, 2005. Disponível em: http://www.scielo.br/pdf/cta/v25n4/27632.pdf

MARGRAF, T.; SANTOS, E.N.T.; ANDRADE, E.F., VAN, R.S.M.; GRANATO, D. Effects of geographical origin, variety and farming system on the chemical markers and in vitro antioxidant capacity of Brazilian purple grape juices. Food Research International, v. $82, \quad$ n. $\quad 1$, p. 145-155. 2016. Disponível em: https://www.sciencedirect.com/science/article/pii/S0963996916300485

MELO, G.C.; ARCANJO, D.R.; LIMA, A.V.S.; SANTOS, M.R.L. Qualidade microbiológica e aceitabilidade de néctar de uva light e tradicional. V Congresso Estadual de Iniciação Científica e Tecnológica do IF Goiano. Campus Iporá. 2016. Disponível em: https://even3.azureedge.net/anais/33788.pdf

OLSSON, E.; MENZEL, C.; JOHANSSON, C.; ANDERSSON, R.; KOCH, K.; JÄRNSTRÖM, L. The effect of pH on hydrolysis, cross-linking and barrier properties of starch barriers containing citric acid. Carbohydrate Polymers, v. 98, n. 2, p. 1505-1513, 2013. Disponível em: https://www.ncbi.nlm.nih.gov/pubmed/24053833

OSMAN, H.E.; MAALEJ, N.; SHANMUGANAYAGAM, D.; FOLTS, J.D. Grape juice but not orange or grapefruit juice inhibits platelet activity in dogs and monkeys (Macaca fasciularis). Journal Agriculture and Food Chemistry, v. 128, n. 2, p. 2307-2312, 1998. Disponível em: https://www.ncbi.nlm.nih.gov/pubmed/9868174

POLONIO, N.C.V.; ROCHA, C.L.M.S.C.; CLEMENTE, E. Trans-resveratrol concentrations and antimutagenic potential of juice from the grape cultivars Vênus. Genetics and Molecular Research, v.13, n.1, p.1152-1159, 2014. Disponível em: https://pdfs.semanticscholar.org/2c65/bad227aa33af39116f49ebc53432f09ae955.pdf

PONTES, P.R.B.; SANTIAG0, S.S.; SZABO, T.N.; TOLEDO, L.P.; GOLLÜCKE, A.P.B. Atributos sensoriais e aceitação de sucos de uva comerciais: Sensory attributes and acceptance of commercial grape juices. Ciência e Tecnologia de Alimentos, v. 2, n. 30, p. 313-318. 2010. Disponível em: http://www.scielo.br/pdf/cta/v30n2/04.pdf

RIZZ0N, L.A; MANFROI, V; MENEGUZZ0, J. Elaboração de suco de uva na propriedade vitícola. Bento Gonçalves: Embrapa Uva e Vinho, v. 21, n. $1, \quad$ p. $\quad 7-21, \quad 1998 . \quad$ Disponível em: https://ainfo.cnptia.embrapa.br/digital/bitstream/item/26032/1/Doc2l.pdf

RODRIGUES, R.F.C.; LIMA, A.; MELO, A.C.F.L.; TRINDADE, R.A. Physicochemical characterisation, bioactive compounds and in vitro antioxidant activities of commercial integral grape juices. International Food Research Journal, v. 29 , n. 2, p. 469-479, 2019. Disponível em: http://www.ifrj.upm.edu.my/26\%20(02)\%202019/(12).pdf

ROLDAN, B.B. Influência do método de extração sobre a composição química de suco de uva bordô (Vitis labrusca). 57 f. Dissertação (Mestrado) - Curso de Programa de Pós-graduação em Ciência e Tecnologia de Alimentos, Instituto de Ciência e Tecnologia de Alimentos, Universidade Federal do Rio Grande do Sul, Porto Alegre, 2016. Disponível em: https://www.lume.ufrgs.br/bitstream/handle/10183/150292/001008524.pdf?sequence=1

SINGLETARY, K.W.; STANSBURY, M.J.; GIUSTI, M.; VAN BREEMEN, R.B.; WALLIG, M.; RIMANDO, A. Inhibition of rat tumorigenesis by concord grape juice constituents, Journal of Agricultural and Food Chemistry, v. 51, p. 7280 -7286, 2003. Disponível em: https://pubs.acs.org/doi/10.1021/jf0302781

SINGLETON, V.L.; ROSSI, J.A. Colorimetry of total phenolics with phosphomolybdicphosphotungstic acid reagents. American Journal of Enology and Viticulture, v. 16, p.144-158, 1965. Disponível em: https://www.ajevonline.org/content/16/3/144

SOUZA, A.V.; VIEIRA, M.R.S.; PUTTI, F.F. Correlations between the phenolic compounds and antioxidant activity in the skin and pulp of table grape varieties. Brazilian Journal of Food Science and Technology, v. 21, n. 1, p.1-6, 2018. Disponível em: http://www.scielo.br/pdf/bjft/v21/1981-6723-bjft-21-e2017103.pdf

TAC0. Tabela brasileira de composição de alimentos. NEPA - Unicamp, p.161, 2011.Disponível em:https://www.cfn.org.br/wp-content/uploads/2017/03/taco_4_edicao_ampliada_e_revisada.pdf 
TOALDO, I.M.; CRUZ, F.A.; ALVES, T.L.; GOIS, J.S.; BORGES, D.L.; CUNHA, H.P.; SILVA, E.L.; BORDIGNON, L.M.T. Bioactive potential of Vitis labrusca L. grape juices from the Southern Region of Brazil: Phenolic and elemental composition and effect on lipid peroxidation in healthy subjects. Food Chemistry, v. 173, n. 1, p. 527-535, 2015. Disponível em: https://www.ncbi.nlm.nih.gov/pubmed/25466055

VARGAS, P.N.; HOELZEL, S.C.; ROSA, C.S. Determinação do teor de polifenóis totais e atividade antioxidante em sucos de uva comerciais. Alimentos e Nutrição, v. 19, n. 1, p.11-15, 2008. Disponível em: http://servbib.fcfar.unesp.br/seer/index.php/alimentos/article/viewFile/194/199

YAMAGUCHI, T.; TAKAMURA, H.; MATOBA, T.; TERA0, J. HPLC method for evaluation of the free radical scavenging activity of foods by using 1,1-Diphenyl-2picrylhydrazyl. Bioscience, Biotechnology, and Biochemistry, v. 62, n.6, p. 1201-1204, 1998. Disponível em: https://www.ncbi.nlm.nih.gov/pubmed/9692204 